\title{
Explorando o Uso dos Elementos do LOM na Determinação dos Aspectos de Qualidade dos Objetos de Aprendizagem a partir de Critérios de Qualidade de Informação
}

\author{
Daniel Lichtnow ${ }^{1}$, Isabela Gasparini ${ }^{2}$, Verlani Timm Hinz ${ }^{3,4}$, Marcelo S. Pimenta ${ }^{4}$, \\ José Palazzo Moreira de Oliveira ${ }^{4}$ \\ ${ }^{1}$ Colégio Politécnico - Universidade Federal de Santa Maria (UFSM) \\ Av. Roraima, $\mathrm{n}^{\circ}$ 1000, Campus UFSM - 97.105-900- Santa Maria - RS - Brasil \\ ${ }^{2}$ Centro de Ciências Tecnológicas- Universidade do Estado de Santa Catarina (UDESC) \\ Campus Prof. Avelino Marcante S/N - 89.223-100 - Joinville - SC - Brasil \\ ${ }^{3}$ Instituto Federal Sul-Rio-Grandense - Pelotas-Visconde da Graça (IFSUL/CAVG) \\ Av. Ildefonso Simões Lopes, n²791 - 96.060-290 - Pelotas - RS - Brasil \\ ${ }^{4}$ Instituto de Informática - Universidade Federal do Rio Grande do Sul (UFRGS) \\ Caixa Postal 15.064 - 91.501-970 - Porto Alegre - RS - Brasil \\ dlichtnow@politecnico.ufsm.br, isabela.gasparini@udesc.br, \\ verlanitimm@cavg.ifsul.edu.br, \{mpimenta, palazzo\}@inf.ufrgs.br
}

\begin{abstract}
The present work propose to evaluate the learning object quality using the LOM - Learning Object Metadata and data quality criteria. In this work, for each data quality dimension are identified LOM elements that can be used as quality indicators. The aim is to become easier the identification of learning objects to will be recommended for users.
\end{abstract}

Resumo. O presente trabalho propõe avaliar a qualidade de um objeto de aprendizagem a partir de elementos do padrão LOM - Learning Object Metadata e de critérios utilizados para avaliação da qualidade de dados. Neste trabalho, para cada dimensão de qualidade considerada são identificados elementos LOM que podem servir como indicadores de qualidade. $O$ objetivo é facilitar a identificação dos objetos de aprendizagem a serem recomendados para usuários.

\section{Introdução}

Objetos de aprendizagem (OA) podem ser definidos como "qualquer entidade, digital ou não digital, que possa ser utilizada, reutilizada ou referenciada durante o aprendizado suportado por tecnologias" [IEEE 2002]. Um problema recorrente é identificar OAs que tenham a qualidade adequada para uso em um contexto específico. Visando facilitar a identificação de OAs úteis para os usuários, vários sistemas de recomendação vem sendo criados. Sistemas de recomendação são sistemas capazes de auxiliar no processo de indicar ou selecionar itens (OAs, por exemplo) úteis para um determinado usuário ou grupo de usuários [Resnick e Varian 1997]. Exemplos de sistemas de recomendação voltados para a área de Educação a Distância (EAD) são [Zaina et al. 2012], [Primo et 
al. 2010], [Ochoa e Duval 2006] [Ferreira e Raabe 2009] [Cho et al. 2007], [Anderson et al. 2003] e [Casagrande et al. 2013].

Dentre as abordagens possíveis para criação de sistemas de recomendação está a baseada em conteúdo. A abordagem baseada em conteúdo consiste em avaliar a similaridade de um item com o perfil de interesses de um usuário [Adomavicius e Tuzhilin, 2005]. Podemos citar os trabalhos de [Zhu et al. 2007], [Zhuhadar et al. 2009] e [Mooney e Roy 2000], como exemplos de implementação de sistemas de recomendação que utilizam a abordagem baseada em conteúdo para sugerir e auxiliar o usuário na identificação de OAs que possam ser úteis para a realização de alguma atividade didática.

$\mathrm{Na}$ abordagem baseada em conteúdo, a qualidade da recomendação produzida é influenciada pela descrição dos itens. Para descrever um OA podem ser usados metadados. Metadados podem ser definidos como dados sobre os dados e vem sendo utilizados para descrever vários tipos de recursos (arquivos MP3, livros, Web sites, imagens e bancos de dados ${ }^{1}$ ). No caso dos OAs, várias iniciativas de padronização dos seus metadados foram feitas e vem sendo consideradas na construção de sistemas de recomendação [Primo et al. 2010] [Casagrande et al. 2013]. Dentre as iniciativas de padronização dos metadados para descrição dos OAs estão, por exemplo, o padrão LOM (Learning Object Metadata) [IEEE, 2002] e o padrão OBAA (Objetos de Aprendizagem Baseados em Agentes) [OBAA 2011], sendo que o OBAA acrescenta elementos ao LOM.

No presente trabalho, o objetivo está em explorar o quanto os metadados definidos no $L O M$ podem avaliar diferentes aspectos de qualidade de um OA. Neste sentido, considerando que OAs fornecem aos seus usuários informações úteis para o seu aprendizado, os diferentes aspectos de qualidade considerados no trabalho consistem de critérios de qualidade utilizados na avaliação de dados/informações. Relacionar metadados a critérios de qualidade de dados/informações ou mesmo avaliar OAs a partir destes critérios não é algo novo [Naumann e Rolker 1999] [Gasparini et al., 2009]. No entanto, desconhecemos trabalhos que realizaram esta análise a partir de elementos do $L O M$.

Inicialmente na seção 2 é apresentada a metodologia de desenvolvimento do trabalho. Na seção 3 é feita uma breve descrição do $L O M$ e também dos critérios de qualidade de dados usados como base para o desenvolvimento do trabalho. A seção 4 analisa os elementos do $L O M$ que podem ser utilizados na avaliação dos critérios de qualidade considerados no trabalho. Já a seção 5 mostra alguns cenários de uso que permitem vislumbrar a aplicação prática destes critérios. A seção 6 faz uma análise das possibilidades geradas a partir do trabalho. Finalmente, a seção 7 apresenta as considerações finais.

\section{Metodologia}

O trabalho começou com a análise das dimensões de qualidade de dados/informações, sendo que o principal trabalho considerado foi [Wang e Strong 1996], por ser um dos

\footnotetext{
${ }^{1}$ http://dublincore.org/metadata-basics/
} 
mais referenciados trabalhos sobre qualidade de dados/informações. Em seguida, realizou-se a análise dos elementos de metadados definidos pelo LOM. Os elementos de metadados definidos no $L O M$ foram então associados às dimensões de qualidade consideradas no trabalho, sendo discutidas formas de utilizar estes como indicadores de qualidade.

\section{Referencial Teórico}

Esta seção descreve sucintamente alguns aspectos relacionados ao $L O M$ e as dimensões de qualidade de dados/informações consideradas no trabalho.

\subsection{LOM - Learning Object Metadata}

O padrão $L O M$ consiste de uma hierarquia de elementos de metadados. Estes elementos estão agrupados em nove categorias. Uma descrição detalhada destes elementos está fora do escopo do trabalho e o espaço não permite, detalhes podem ser consultados em [IEEE 2002]. Comentários sobre os elementos são feitos na seção 4.

\subsection{Qualidade de dados/informações}

A definição de qualidade para produtos e serviços feita por Juran (1962) relaciona qualidade à adequação para uso - "fitness for use" e vem sendo utilizada em diversos contextos, incluindo trabalhos que lidam com qualidade de dados. Dentre os trabalhos que discutem critérios de qualidade de dados um dos mais referenciados é o de [Wang e Strong, 1996].

Wang e Strong (1996) usam o termo dimensão para representar um aspecto específico de qualidade e agrupam estas dimensões em 4 categorias (Figura 1):

1. Intrínseca - Intrinsic. Dimensões de qualidade que são avaliadas independente temente do contexto e das preferências do usuário (consideram especialmente a correção e consistência dos dados);

2. Contextual - Contextual. Dimensões de qualidade que devem ser avaliadas a partir de um determinado contexto (consideram o perfil e necessidades do consumidor dos dados);

3. Representacional - Representational. Dimensões de qualidade que enfatizam o formato e a facilidade de processamento dos dados;

4. Acessibilidade - Accessibility. Dimensões de qualidade que enfatizam a disponibilidade, facilidade de acesso e segurança dos dados.

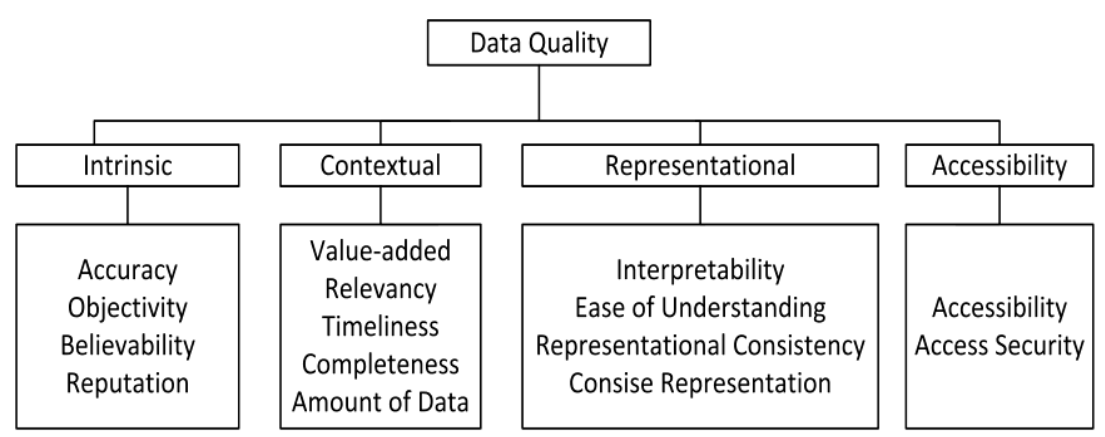

Figura 1. Dimensões - qualidade de dados/informações [Wang e Strong, 1996] 
Na seção 4 são apresentadas considerações sobre cada uma das dimensões de qualidade presentes na Figura 1. Cabe ressaltar que alguns trabalhos fazem a distinção entre dados e informação, destacando que informação deriva dos dados, porém, neste trabalho (como em outros) não é feita tal distinção.

\section{Dimensões de Qualidade de Dados/Informações e Elementos LOM}

Para mensurar cada dimensão de qualidade (i.e. cada aspecto específico de qualidade) é necessário fazer uso de um conjunto de indicadores que estão relacionados a um OA. Conforme já salientado, no presente trabalho estes indicadores correspondem a elementos LOM. Inicialmente, são descritas as dimensões de qualidade mostradas na seção 3, sendo observadas as definições feitas em [Wang e Strong, 1996]. Após, são identificados elementos do LOM que servem como indicadores para as dimensões (alguns indicadores de qualidade podem estar relacionados a uma ou mais dimensões de qualidade).

Acurácia - Accuracy. Esta dimensão está relacionada a aspectos de correção dos dados e a ausência de erros. Verificar se um dado está correto não é uma tarefa trivial. Uma possibilidade seria comparar os dados/informações presentes em um OA com aqueles presentes em uma fonte confiável. Evidentemente não é fácil identificar uma fonte confiável disponível. Além disto, para dados não estruturados (vídeo, texto, etc.) esta comparação assume uma complexidade ainda maior do que para dados estruturados. Assim, uma possibilidade é considerar que alguns elementos do LOM podem, indiretamente, indicar acurácia. Estes elementos são 2.1 Version e 2.2 Status. No caso do elemento 2.1 Version é possível estimar que quanto maior o valor a ele associado, maior a acurácia (especialmente no caso das diferentes versões de um mesmo OA). Já o caso do elemento 2.2 Status, que pode ser preenchido com os valores "draft", "final", "revised", "unavailable" (valores discretos em uma escala ordinal) é possível concluir que um OA com status revised possui, em princípio, maior acurácia do que um OA com status draft ou mesmo final, assumindo que o processo de revisão garante maior acurácia.

Credibilidade - Believability. Credibilidade e reputação - reputation podem ser combinadas em uma só dimensão, sendo aqui mantida apenas credibilidade, uma vez que tanto credibilidade quanto reputação estão relacionadas à autoria do conteúdo ou ao fornecedor da informação [Naumann et al. 1999]. Assim, um indicador importante para credibilidade é a informação sobre o criador do OA (a credibilidade dada ao criador do OA é estendida ao próprio OA). Um opção é realizar a avaliação da credibilidade usando notas atribuídas explicitamente pelos usuários [Naumann et al. 1999]. Esta avaliação pode ainda ser implícita - um grande número de referências a um OA na Web, por exemplo, pode servir como indicador.

No caso dos elementos do $L O M$, possíveis indicadores de credibilidade são 2.3 Contribute e 4.3 Location. O elemento 2.3 Contribute, está relacionado a entidades (pessoas e organizações, por exemplo) que contribuíram com o estado do OA durante seu ciclo de vida (criação, edição e/ou publicação). Desta forma, uma possibilidade é analisar a reputação das entidades envolvidas. Evidentemente automatizar esta avaliação não é fácil, sendo uma possibilidade é verificar o número (e o uso) de OAs criados por um Contribute que estão dentro de um Ambiente Virtual de Aprendizagem (AVA) ou 
dentro de um repositório digital. Já o elemento 4.3 Location indica a localização do objeto de aprendizagem (URL) abrindo assim a possibilidade de verificar o número de referências ao OA na Web e até mesmo o Pagerank relacionado à $U R L$.

Objetividade - Objectivity. Esta dimensão está relacionada ao grau de imparcialidade dos dados, uma vez que dados podem ser influenciados pelas preferências ou intenções do criador/fornecedor [Bizer 2007]. Uma possibilidade é realizar alguma análise no estilo de escrita usado no atributo 1.4 Description. Cabe salientar que a Wikipedia adota alguns critérios de forma a manter a neutralidade dos seus artigos (a presença de adjetivos na descrição do objeto indica falta de neutralidade, i.e. um menor grau de objetividade ${ }^{2}$ ).

Tempestividade - Timeliness. A idade de um dado deve ser adequada, considerando seu contexto de uso. Um elemento que claramente deve ser utilizado é o elemento 2.3.3 Date. Cabe também ressaltar que para alguns OAs, a idade é um fator mais crítico: um OA relacionado ao ensino de HTML, criado em 13/03/1997, não é (provavelmente) dos mais indicados.

Relevância - Relevancy. Esta dimensão ressalta o fato de que o dado deve ser útil para a realização de uma determinada tarefa. Elementos de metadados que descrevem o conteúdo de um OA são úteis para determinar sua relevância. No LOM estes elementos são 1.2 Title, 1.4 Description e 1.5 Keyword. Uma possibilidade envolve o uso de métricas utilizadas em Recuperação de Informação como o cosine que permite comparar documentos [Salton e McGill, 1983]. Assim, a partir de uma consulta ou de termos associados ao perfil do usuário, o sistema pode verificar quais OAs são adequados.

Completeza - Completeness. Nesta dimensão é verificado o quanto os dados são suficientemente abrangentes considerando às necessidades de um usuário. Assim, no caso de um OA é necessário verificar se ele atende todas as necessidades do usuário. No contexto dos bancos de dados relacionais, o grau de completeza pode ser medido observando quantos campos de uma tupla de uma tabela não possuem valor e deveriam ter [Batini et al, 2009]. No caso de um OA, elementos como 1.2 Title, 1.4 Description e 1.5 Keyword considerados como indicadores para relevância são úteis aqui. Porém, deve ser verificado se o OA atende necessidades específicas de um usuário. Assim, se o usuário deseja aprender sobre CSS e $H T M L$, por exemplo, um OA completo é um que aborde os dois temas.

Valor Adicionado - Value-added. Uma possibilidade aqui é considerar que um OA muito similar a outro já utilizado não seja de grande valor para um usuário. Aqui cabe observar que os sistemas de recomendação que usam a abordagem baseada em conteúdo costumam ter problemas (super especialização) por recomendarem sempre mais do mesmo (i.e. é recomendado sempre algo similar ao que um usuário já avaliou como bom no passado) [Adomavicius e Tuzhilin, 2005]. Para verificar se um objeto de aprendizagem é muito similar a outro uma possibilidade é comparar a descrição contida nos elementos 1.2 Title, 1.4 Description e 1.5 Keyword. O desafio aqui está em identificar objetos de aprendizagem relacionados ao mesmo tema, mas que não sejam

\footnotetext{
${ }^{2}$ http://pt.wikipedia.org/wiki/Wikip\%C3\%A9dia:Princ\%C3\%ADpio_da_imparcialidade\#Adjetivos
} 
muito similares. Neste sentido uma possibilidade seria recomendar objetos de aprendizagem que possuam as mesmas palavras-chave (1.5 Keywords), mas título (1.2 Title) e descrição (1.4 Description) diferentes (em algum grau). Nota-se que um interessante tema de pesquisa é o estudo de como combinar Relevância, Valor Adicionado e Completeza.

Volume de Dados - Amount of Data. O volume de dados deve ser adequado às necessidades do usuário. Os elementos 4.7 Duration (indica a duração de um vídeo ou arquivo de áudio) e 5.9 Typical Learning Time (tempo aproximado que os usuários irão gastar para utilizar o objeto de aprendizagem) podem eventualmente ser considerados aqui. Além destes, o atributo 4.2 Size que indica o tamanho de um objeto de aprendizagem em bytes, pode ser considerado.

Interpretabilidade - Interpretability. O principal aspecto considerado aqui é verificar o quanto os dados estão em uma linguagem apropriada. Bizer (2007) salienta que algumas "tecnologias Web procuram aumentar a interpretabilidade", neste contexto estão às chamadas schema languages (XML schema, por exemplo) e linguagens relacionadas à Web Semântica. Assim, o elemento do LOM a ser utilizado é 4.1 Format que identifica o software necessário para acessar o OA.

Compreensibilidade - Ease of understanding. Seguindo esta dimensão é avaliado o quanto os dados são facilmente compreendidos. É associada à interpretabilidade [Bizer, 2007]. Porém enquanto Interpretabilidade faz referência ao fato da informação ser representada em uma notação apropriada, compreensibilidade faz referência à capacidade subjetiva de um indivíduo compreender a informação [Naumann et al., 1999]. Os elementos LOM que podem ser considerados indicadores para esta dimensão de qualidade são 5.5 Intended End User Role ("teacher", "author", "learner", etc.), 5.6 Context (qual o contexto de uso do objeto "school", "higher education", "training”, etc.), 5.7 Typical Learning Time , 5.8 Difficulty("very easy", "easy", "medium", "difficult", "very difficult") e 5.11 Language.

Consistência Representacional - Representational Consistency. Enfatiza a necessidade dos dados serem representados em formato adequado. Uma possibilidade é considerar o uso de padrões na construção do OA - avaliar se ele é disponibilizado no formato Sharable Content Object Reference Model (SCORM), por exemplo.

Representação Concisa - ConciseRepresentation. Enfatiza o grau em que a informação é apresentada de forma compacta e direta. Esta dimensão está relacionada a duas dimensões de qualidade complementares: Volume de Dados e Completeza. O elemento do LOM que está relacionado a esta dimensão de qualidade é 5.4 Semantic Density. Aqui, conforme exposto em [IEEE 2002], a questão não é a dificuldade, estando relacionada a questões de duração de um vídeo, por exemplo. Portanto elementos usados para avaliar o volume de dados são também relevantes aqui.

Acessibilidade - Accessibility. Elementos LOM que permitem avaliar acessibilidade são 4.1 Format, 4.4. Requirements (especialmente o sub-elemento 4.4.1.1 Type que destaca quais são os requisitos de hardware, software e rede para uso do OA). Existe ainda o elemento 4.6 Other Plataform Requirements, e o elemento 5.1 Interactivity Type que descreve o tipo de interação com o objeto de aprendizagem ("active", "expositive" e "mixed"). 
Segurança de Acesso - Access Security. Aspectos de segurança não são normalmente uma preocupação na criação de um OA, porém, o elemento 9.1 Purpose do $L O M$ que trata do objetivo de classificar um $\mathrm{OA}$, pode ter um valor sobre o nível de segurança do OA.

\section{Cenários de Uso}

A partir da definiç̧ão de indicadores de qualidade para as dimensões de qualidade dos dados/informações é possível analisar sua aplicação na identificação de OAs úteis para usuários. Em primeiro lugar é necessário caracterizar o contexto de uso do OA. Contexto, neste trabalho, refere-se a "qualquer informação que pode ser usada para caracterizar a situação de uma entidade (uma pessoa, um lugar ou um objeto) que é considerada relevante para a interação entre o usuário e uma aplicação, incluindo o próprio usuário e a própria aplicação" [Dey 2001]. A seguir, são descritos dois cenários (Figura 2 e Figura 3) que evidenciam o uso destes critérios de qualidade.

Cenário 1. João de 15 anos é aluno de um curso de básico de inglês oferecido na modalidade de EAD. Ele está cursando o ensino médio. Durante a semana ele se desloca de casa para o trabalho e do trabalho para casa usando um ônibus. Este trajeto leva normalmente em torno de 40 minutos. João possui um dispositivo móvel que permite acessar alguns objetos de aprendizagem relacionados a este curso disponíveis em um AVA.
Cenário 2. Ana de 16 anos está cursando o ensino médio. Ana estuda inglês todos os dias após as aulas regulares, juntamente com um grupo de alunos, na biblioteca da escola, através de um repositório de materiais online gratuito. Ana estuda durante 1 hora. Ela utiliza o computador da escola.

Figura 2. Descrição dos Cenários

No Cenário 1 apresentado na Figura 2, algumas dimensões de qualidade de dados/informações assumem especial importância: Relevância, Volume de Dados, Compreensibilidade e Acessibilidade. No que se refere à relevância, o $\mathrm{OA}$ a ser acessado deverá tratar do assunto de estudo (Verbos em Inglês no Presente). No que se refere ao volume de dados será preciso considerar 4.7 Duration (não mais de 40 minutos) e 5.9 Typical Learning Time (não mais de 40 minutos). Já com respeito a compreensibilidade 5.5 IntendedEndUser Role deve indicar "learner" e 5.8 Difficulty deve ser "very easy", "easy" ou “medium”). Já com relação à acessibilidade devem ser recomendados apenas OAs que possam ser executados na plataforma que João possui (Um smartphone com Android). Além disto, como ele está no ônibus não é desejável que o elemento 5.1 Interactivity Type indique "active", dado a dificuldade de interação durante o deslocamento do ônibus.

O Cenário 2 da Figura 2, apresenta alguns aspectos bastante distintos do Cenário 1. A dimensão relevância permanece sendo importante. No que se refere ao volume de dados será preciso também considerar 4.7 Duration (não mais de 1 hora) e 5.9 Typical Learning Time (não mais de 1 hora). Em relação à compreensibilidade 5.5 Intended End User Role deve indicar "learner" e 5.8 Difficulty deve ser "very easy", "easy" 
ou "medium". Já no que diz respeito à acessibilidade, Ana não tem as mesmas restrições de João (um OA com 5.1 Interactivity Type indicando "active" não gera problema). Porém, como Ana acessa OAs em um repositório e não em um AVA como João, é necessário levar em conta de forma mais efetiva algumas dimensões intrínsecas. Se um material em um AVA é colocado pelo professor, é possível assumir que este material atende os requisitos das dimensões de qualidade classificados como intrínsecas (acurácia e objetividade). Neste sentido, considerar estes critérios de qualidade é, em princípio, mais necessário quando está se buscando um OA em um repositório público, em que diversos materiais são colocados por diferentes pessoas.

\section{Análise das Contribuições}

Os critérios de qualidade para dados/informações consideram que qualidade significa adequação para uso [Juran 1962], o que enfatiza a necessidade de considerar o contexto. A mesma preocupação com o contexto está presente em sistemas de recomendação [Adomavicius e Tuzhilin, 2005]. O uso de critérios de qualidade para dados/informações evidencia ainda a necessidade de que a qualidade seja avaliada usando múltiplos critérios (dimensões).

Os cenários apresentados demonstram que algumas dimensões de qualidade de dados/informações podem assumir maior importância em determinado contexto. Um exemplo é a questão da acessibilidade apresentada no Cenário 1, que para o aluno João é considerada muito relevante, visto que ele está acessando o ambiente de um dispositivo móvel. Porém, para a aluna Ana, do Cenário 2, esse aspecto não é tão essencial visto que ela está utilizando o computador na escola.

Uma possibilidade de uso da proposta apresentada é refinar o resultado de um processo de recomendação tradicional (que use abordagem baseada em conteúdo ou colaborativa) considerando as dimensões de qualidade e indicadores identificados no trabalho. Proposta similar é feita em [Primo et al. 2010] onde os metadados OBAA são usados para refinar a recomendação considerando o perfil do usuário (perfil cognitivo, por exemplo). Assim, a recomendação pode ter como resultado, além de uma lista de OAs a serem explorados, uma explanação sobre como cada OA recomendado atende a diferentes critérios de qualidade (um dashboard dos critérios de qualidade). Além disso, algumas dimensões de qualidade de dados/informações enfatizam aspectos desconsiderados em sistemas de recomendação de OAs, como por exemplo a Acurácia.

O uso de alguns indicadores identificados no trabalho pode ainda auxiliar na resolução de problemas presentes nas abordagens tradicionais dos sistemas de recomendação. Já foi citada a super especialização como problema na abordagem baseada em conteúdo (e.g. ver Valor Adicionado na seção 4). Um problema na abordagem colaborativa é o de que um novo item não será recomendado por não ter sido avaliado por nenhum usuário (cold start) [Adomavicius e Tuzhilin, 2005]. Fazendo uso dos indicadores relacionados à credibilidade seria possível assumir que um novo OA criado por um mesmo Contribute (elemento LOM 2.3 Contribute) que teve vários OAs bem avaliados será também bem avaliado. 


\section{Considerações Finais}

À medida que os ambientes virtuais de aprendizagem se tornam mais sofisticados, novas oportunidades e desafios emergem, como a recomendação de OAs em diferentes cenários. No presente trabalhou foi proposta a avaliação de OAs por meio dos seus metadados, seguindo o padrão estabelecido no $L O M$ e o uso de critérios de qualidade usados para avaliar dados/informações. O objetivo foi estabelecer uma forma mais criteriosa de avaliação dos OAs que possa auxiliar na implementação de sistemas de recomendação, tentando contribuir no melhoramento do processo de ensinoaprendizagem, através da recomendação dos melhores e mais apropriados OAs. Trabalhos futuros envolvem o uso efetivo destes critérios em sistemas de recomendação presentes em AVAs e repositórios de OAs, a análises das recomendações realizadas e sua contribuição no processo de ensino-aprendizagem.

\section{Agradecimentos}

Este trabalho foi parcialmente apoiado pelo CNPq (Conselho Nacional de Desenvolvimento Científico e Tecnológico) e pela CAPES (Coordenação de Aperfeiçoamento de Pessoal de Nível Superior).

\section{Referências}

Adomavicius, G.; Tuzhilin, A. (2005). Toward the next generation of recommender systems: A survey of the state-of-the-art and possible extensions. IEEE Transactions on Knowledge and Data Engineering, Piscataway, 17(6), p. 734-749.

Anderson, M. et al. (2003). RACOFI: A Rule-Applying Collaborative Filtering System. In Proc. IEEE/WIC COLA'03.

Batini, C.; Cappiello, C.; Francalanci, C.; Maurino, A. (2009). Methodologies for data quality assessment and improvement. ACM Computing Surveys (CSUR), 41(3).

Bizer, C. (2007). Quality Driven Information Filtering: In the Context of Web Based Information Systems. VDM Publishing.

Casagrande, M. F. R.; Kozima, G.; Willrich, R. (2013) Técnica de Recomendação Baseada em Metadados para Repositórios Digitais Voltados ao Ensino In: Simpósio Brasileiro de Informática na Educação (SBIE), p.677-686.

Cho, et al. (2007). Collaborative filtering using dual information sources. IEEE Intelligent Systems, 26(3).

Dey, A. K. (2001). Understanding and using context. Personal and ubiquitous computing, 5(1), 4-7.

Ferreira, V. H.; Raabe, A. L. A. (2010). LORSys - Um Sistema de Recomendação de Objetos de Aprendizagem SCORM, Revista Novas Tecnologias na Educação (RENOTE), 8(2),1679-1916.

Gasparini, I.; Lichtnow, D.; Pimenta, M. S.; Palazzo M. de Oliveira, J. (2009). Quality ontology for recommendation in an adaptive educational system. In International Conference on Collaborative Systems, p. 329-334. 
IEEE (2002). Draft Standard for Learning Object Metadata, Disponível em: http://grouper.ieee.org/groups/ltsc/wg12/20020612-Final-LOM-Draft.html. Acesso em 10 Junho de 2014.

Juran, J. M. (1962). Quality control handbook. In Quality control handbook. New York: McGraw-Hill.

Mooney R.; Roy L. (2000). Content-based book recommending using learning for text categorization. In Fifth ACM Conference on Digital Libaries, pp. 195-204.

Naumann, F.; Leser, U.; Freytag, J. C. (1999). Quality-driven Integration of Heterogenous Information Systems. In Conferences on Very Large Databases, $p$. 447-458.

Naumann, F.; Rolker, C. (1999) Do metadata models meet IQ requirements. In: 4th Conference on Information Quality IQ, p.99-114.

Ochoa, X.; Duval, E. (2006). Use of contextualized attention metadata for ranking and recommending learning objects. In: Workshop on Contextualized attention metadata: collecting, managing and exploiting of rich usage information, p. 9-16.

OBAA (2011). Portal OBAA, Disponível em: http://www.portalobaa.org/obaac, Acesso em: 10 de Junho de 2014.

Primo, T. T.; Vicari, R. M.; da Silva, J. M. C. (2010). Rumo ao uso de metadados educacionais em sistemas de recomendação. In: Simpósio Brasileiro de Informática na Educação (SBIE).

Resnick, P.; Varian, H. R. (1997). Recommender systems. Communications of ACM, 40(3),56-58.

Salton, G.; McGill, M. J. (1983). Introduction to modern information retrieval. New York: McGrill-Hill.

Wang, R. Y.; Strong, D. M. (1996) Beyond accuracy: what data quality means to data consumers. Journal of Management Information System, 12(4), 5-33.

Zaina, L. A.; Bressan, G.; Cardieri, M. A. A.; Rodrigues Júnior, J. F. (2012). E-lors: Uma abordagem para recomendacao de objetos de aprendizagem. Revista Brasileira de Informática na Educação (RBIE), 20(1).

Zhu, F., Ip; H. H.-S.; Fok, A.W. P.; Cao, J. (2007). Peres: A personalized recommendation education system based on multi-agents \& Scorm. In: Advances in Web Based Learning - ICWL, LNCS, v 4823, p. 31-42.

Zhuhadar, L.; Nasraoui, O.; Wyatt, R.; Romero, E. (2009). Multi-model ontology-based hybrid recommender system in e-learning domain. In: Web Intelligence and Intelligent Agent Technologies, p. $91-95$. 